\title{
Minimal model of torsion mediated dark matter
}

\author{
Basabendu Barman $\odot,{ }^{1, *}$ Tapobroto Bhanja $\odot,{ }^{1, \dagger}$ Debottam Das, ${ }^{2, \hbar}$ and Debaprasad Maity ${ }^{1, \S}$ \\ ${ }^{1}$ Department of Physics, Indian Institute of Technology Guwahati, Assam 781039, India \\ ${ }_{2}^{2}$ Institute of Physics, Bhubhaneswar, Odhisha-751005, India \\ and Homi Bhabha National Institute, Training School Complex, Anushakti Nagar, Mumbai 400085, India
}

(Received 2 January 2020; accepted 11 March 2020; published 10 April 2020)

\begin{abstract}
We present a minimal model of fermionic dark matter (DM), where a singlet Dirac fermion can interact with the Standard Model (SM) particles via the torsion field of gravitational origin. In general, torsion can be realized as an antisymmetric part of the affine connection associated with the spacetime diffeomorphism symmetry and thus can be thought of as a massive axial vector field. Because of its gravitational origin, the torsion field couples to all the fermion fields including the DM with equal strength, which makes the model quite predictive. The DM is naturally stable without any imposition of ad hoc symmetry, e.g., $\mathcal{Z}_{2}$. Apart from producing the correct thermal abundance, a singlet fermion can easily evade the stringent bounds on the spin-independent DM-nucleon direct detection cross section due to its axial nature. However, in the allowed parameter space, strong bounds can be placed on the torsion mass and its couplings to fermions from the recent LHC searches. Assuming a nonuniversal torsion-DM and torsion-SM coupling, smaller values of torsion masses may become allowed. In both cases we also study the reach of spin-dependent direct detection searches of the DM.
\end{abstract}

DOI: 10.1103/PhysRevD.101.075017

\section{INTRODUCTION}

The Standard Model (SM) of particle physics in its present form has been extremely successful in accommodating fundamental interactions of nature. However, the model is regarded as incomplete when it comes to explain a few observations, e.g., dark matter (DM) abundance or the matter-antimatter asymmetry in the Universe. In "beyond Standard Model" (BSM) physics, one may explain most of these puzzles in terms of new particles and their couplings with the SM particles. But null results from different accelerator/nonaccelerator based experiments to test these new particles have pushed their masses above $\mathrm{TeV}$ scale.

The presence of DM in the universe has been established via a number of successful experiments through its gravitational impact [1] (for reviews see, e.g., [2-6]), though its nature is yet to be known. One of the most popular and accepted DM candidate is considered to be the WIMP (weakly interacting massive particle), obtained naturally in most of the well motivated BSM scenarios like

\footnotetext{
*bb1988@iitg.ac.in

tapobroto.bhanja@iitg.ac.in

\#debottam@iopb.res.in

§ebu@iitg.ac.in
}

Published by the American Physical Society under the terms of the Creative Commons Attribution 4.0 International license. Further distribution of this work must maintain attribution to the author(s) and the published article's title, journal citation, and DOI. Funded by SCOAP ${ }^{3}$. supersymmetric (SUSY) theories or in the extradimensional models. However, no evidence of this prototype DM candidate has so far been observed in accelerator based experiments like the LHC or in the direct detection (DD) experiments like XENON100 [7], LUX [8], PandaX-II [9] or Xenon-1T [10] which search for DM scatterings off nuclei in detectors. All these searches have essentially put stringent constraints on the parameter space of some of the extensions of the SM (for a review see [11]) like $Z$-portal [12-16], Higgs-portal [17-23] ${ }^{1} Z^{\prime}$-portal [31-39], or pseudoscalar portals [40-42]. Among these models, $Z^{\prime}$-portal with only axial coupling, or pseudoscalar portal models are somewhat preferred for a fermionic DM in particular as nonobservation of any signal, especially in the context of spin-independent (SI) DM-nucleon scattering can be easily accommodated. Driven by the similar quest, here we explore the possibility of DM-SM interactions through the torsion portal which is of geometric origin, thus completely independent of any representation of the grand unified or the SM gauge group.

Due to its geometric origin, the interaction of torsion field with SM has some interesting features. In general, the torsion field can be identified as one of the irreducible representations of the antisymmetric part of the affine connection associated with the spacetime diffeomorphism

\footnotetext{
${ }^{1}$ In the context of SUSY, lightest neutralino DM can efficiently annihilate through Higgs states to accommodate the relic abundance [24-30](for review see [2-4]).
} 
symmetry. Because of its antisymmetric property, the torsion field behaves like a massive axial vector field when it interacts with the SM fields. In a natural extension, one may consider minimal coupling prescription [43] which reduces the dimension of the parameter space of the theory under consideration. ${ }^{2}$ In recent times, a torsion field with masses around $\mathrm{TeV}$ scale has drawn attention in the context of low energy phenomenology [44-51] (for a review see [43]). One of this works also considers a possibility of having torsion as a DM candidate [50] where the DMs are pair annihilated through Higgs portal. Their analysis require vanishingly small coupling between the torsion and matter field.

In the present work, we consider a minimal model of a fermionic DM in presence of external propagating torsion field, where torsion plays the role of a mediator between the dark sector and the visible sector. We ignore any coupling between gauge fields and torsion fields [43] as it may spoil gauge invariance. Since torsion appears in the covariant derivative, the coupling constants with the DM or with the SM fields could be identical and independent of their intrinsic properties such as mass, color, flavor, charge, etc. This particular property makes our model economical though tightly constrained from different experimental observations, particularly from the LHC searches. ${ }^{3}$ Assuming a minimal deviation, i.e., allowing a nonequal torsion-DM coupling compared to the SM fermions, we find that LHC constraints can be easily accommodated. To this end we may note a few important points:

(i) Stability of the DM does not require imposition of any ad-hoc symmetry (like $\mathcal{Z}_{2}$ or $\mathcal{Z}_{3}$ symmetry in general). Since there is no renormalizable coupling between DM-SM, the decay of DM to SM particles is automatically forbidden.

(ii) The presence of the axial coupling in the DM-SM interaction ensures spin-dependent (SD) direct search cross section. In this sense, stringent limits from spin independent direct detection experiments on the DM scattering cross section can naturally be evaded.

(iii) The universal coupling $\eta$ does not require any fine tuning, and can assume $\sim O(1)$ value which is compatible with the observed DM relic abundance. In contrast, $\eta \lesssim 10^{-20}$ has been assumed for torsion DM [50] scenario in order to make it stable over the

\footnotetext{
${ }^{2}$ As a matter of fact, superstring theories predict a nonminimal coupling of torsion with the matter fields, such that the compactification of extra-dimensions results in a general theory containing the modified version of the nonminimal couplings at low energy scale.

${ }^{3}$ However the universality in coupling constant can be broken if we consider a High scale model involving torsion. One may need to use renormalization group ( $R G$ ) equations to calculate the coupling constants at the electroweak scale which may be different and may provide with rich phenomenology.
}

cosmological time scale. This forbids any chance to produce such massive vector field at the LHC.

Our present paper is organized as follows. In Sec. II, we recapitulate the essentials of the theory of torsion and their interactions with the fermion fields. Our Sec. III is devoted to the discussion on the experimental and theoretical constraints on the parameter space. In Sec. IV, we study the DM phenomenology of our model with torsion as a mediator between the dark and SM sector. In this analysis we particularly maintain the universality of the torsionfermion (this includes the fermionic DM as well) coupling parameter $\eta$, and discuss the relevant bounds on the torsion mass and its couplings to SM fermions. We further explore the prospects of DM direct detection via a detailed scan over the relic density allowed parameter space. Direct production of the heavy resonances at the LHC may put stringent limits on the universal coupling, which can be evaded if one assumes torsion-DM coupling is different than torsion-SM fermions couplings. It is expected, as the LHC limits are mainly defined for latter interactions. A brief discussion along with changes in DM phenomenology would be presented in Sec. V. Finally, we summarize our key results in Sec. VI.

\section{ORIGIN OF TORSION AND ITS COUPLING WITH MATTER}

We begin this section with a brief introduction to torsion. Here we closely follow Refs. [43-46,49,50]. In the general theory of relativity, the covariant derivative of a vector field $A^{\nu}$ is a tensor and is defined as:

$$
\nabla_{\mu} A^{\nu}=\partial_{\mu} A^{\nu}+\Gamma_{\mu \lambda}^{\nu} A^{\lambda},
$$

where the last term $\Gamma_{\mu \nu}^{\lambda}$ is known as the affine connection. The simplest form of this affine connection in general relativity is known as the Christoffel symbol and is based on the two following special requirements

(i) it is symmetric in nature, $\Gamma_{\mu \nu}^{\lambda}=\Gamma_{\nu \mu}^{\lambda}$,

(ii) the metricity condition i.e., $\nabla_{\alpha} g_{\mu \nu}=0$, where $g_{\mu \nu}$ is the metric tensor of the corresponding spacetime we are working in. When the above two conditions are fulfilled we have an expression of the Christoffel symbol as,

$$
\Gamma_{\mu \nu}^{\lambda}=\frac{1}{2} g^{\lambda \alpha}\left(\partial_{\mu} g_{\nu \alpha}+\partial_{\nu} g_{\mu \alpha}-\partial_{\alpha} g_{\mu \nu}\right)
$$

However, in the Einstein-Cartan extension of general relativity, the said conditions can be relaxed so that the affine connection can have an antisymmetric part defined as:

$$
\tilde{\Gamma}_{\mu \nu}^{\lambda}=\Gamma_{\mu \nu}^{\lambda}+C_{\mu \nu}^{\lambda}
$$

where $C_{\mu \nu}^{\lambda}$ is a mixed tensor field. Thus, new degrees of freedom are introduced into the system which cannot be fixed by the background metric anymore. Therefore, even in 
the flat space limit, this field will survive. A particular irreducible representation of this tensor field may play the role for a torsion field.

For better understanding, we may define the general torsion tensor as

$$
T_{\mu \nu}^{\lambda}=\tilde{\Gamma}_{\mu \nu}^{\lambda}-\tilde{\Gamma}_{\nu \mu}^{\lambda},
$$

which is antisymmetric in its lower indices. The above torsion tensor can be rewritten in terms of irreducible representation:

$$
T_{\mu \nu \lambda}=\frac{1}{3}\left(T_{\nu} g_{\mu \lambda}-T_{\lambda} g_{\mu \nu}\right)-\frac{1}{6} \varepsilon_{\mu \nu \lambda \sigma} S^{\sigma}+q_{\mu \nu \lambda},
$$

where $S^{\lambda}=\varepsilon^{\mu \nu \alpha \lambda} T_{\mu \nu \alpha}$ is torsion trace axial vector mode, $q_{\mu \nu \alpha}$ is tracefree tensor mode satisfying the conditions $q_{\nu \mu}^{\mu}=0$ and $\varepsilon^{\mu \nu \alpha \sigma} q_{\mu \nu \alpha}=0$. The tracefree vector mode is defined as $T_{\mu}=T_{\mu \alpha}^{\alpha}$. Because of its geometric nature and specific parity properties, different irreducible torsion components will have specific type of couplings with the fermions. The general action of a Dirac spinor coupled to torsion field reads:

$\mathcal{S}_{D}=\int d^{4} x\left\{i \bar{f} \gamma^{\mu}\left(\partial_{\mu}-i e A_{\mu}-i \eta_{1} \gamma^{5} S_{\mu}+i \eta_{2} T_{\mu}\right) f-m \bar{f} f\right\}$,

where $\eta_{1}, \eta_{2}$ are nonminimal coupling parameters. Simply from the symmetry properties of various terms of the torsion fields, in the minimal coupling scenario, these parameters may assume fixed values: $\eta_{1}=\frac{1}{8}$ and $\eta_{2}=0$. However, if we consider dynamical torsion interactions with the matter fields, $\eta_{1}$ and $\eta_{2}$ are expected to change under the renormalization group (RG) flow (see, e.g.,[43] and references therein). For phenomenological purposes we choose them as free parameters at low energy scale. Moreover, keeping the simplicity in mind, we will set all the other irreducible components of the torsion field to be zero except the traceless axial vector component $S_{\mu}$.

We consider massive fermion fields in the action mentioned above. In the fermion mass limit $m=0$, the action enjoys an additional gauge symmetry transformation related to torsion:

$$
\begin{aligned}
f \rightarrow f^{\prime} & =f e^{\gamma_{5} \beta(x)}, \quad \bar{f} \rightarrow \bar{f}^{\prime}=\bar{f} e^{\gamma_{5} \beta(x)}, \\
S_{\mu} \rightarrow S_{\mu}^{\prime} & =S_{\mu}-\eta^{-1} \partial_{\mu} \beta(x) .
\end{aligned}
$$

Massive fermion terms may be explained if the said symmetry is softly broken. This in turn will automatically generate the torsion mass term at the loop level, and will be proportional the fermionic mass term. This fact prompts one to consider a massive dynamical torsion field $S_{\mu}$ with the following simplest action (see [43], for details)

$$
\mathcal{S}_{\text {torsion }}=\int d^{4} x\left\{-\frac{1}{4} S_{\mu \nu} S^{\mu \nu}+\frac{1}{2} m_{s}^{2} S_{\mu} S^{\mu}\right\}
$$

where $S_{\mu \nu}=\partial_{\mu} S_{\nu}-\partial_{\nu} S_{\mu}$ and $m_{s}$ is the torsion mass. The first term is the usual Abelian kinetic term which naturally satisfies the above gauge conditions in Eq. (7).

Inclusion of a DM, specifically a SM-singlet Dirac fermion $\psi$, is possible in a similar way since, by construction, the new state interacts with torsion field in the same way like that of a SM fermion. Importantly, the singlet Dirac state cannot have any renormalizable couplings to any SM particles. Thus DM-SM interaction can be only realized through $S_{\mu}$ portal, which reads:

$$
\mathcal{S}_{\mathrm{DM}}=\int d^{4} x\left\{i \bar{\psi} \gamma^{\mu}\left(\partial_{\mu}-i \eta \gamma^{5} S_{\mu}\right) \psi-m_{\psi} \bar{\psi} \psi\right\} .
$$

Finally, the relevant parts of the action which includes the new interactions can be written as:

$$
\begin{aligned}
\mathcal{S}_{\mathrm{New}}= & \mathcal{S}_{\text {torsion }}+\mathcal{S}_{D}+\mathcal{S}_{\mathrm{DM}} \\
= & \int d^{4} x\left\{-\frac{1}{4} S_{\mu \nu} S^{\mu \nu}+\frac{1}{2} m_{s}^{2} S_{\mu} S^{\mu}\right\} \\
& +\int d^{4} x\left[\left\{i \bar{f} \gamma^{\mu}\left(\partial_{\mu}-i \eta_{1} \gamma^{5} S_{\mu}\right) f\right\}\right. \\
& \left.+\left\{i \bar{\psi} \gamma^{\mu}\left(\partial_{\mu}-i \eta \gamma^{5} S_{\mu}\right) \psi-m_{\psi} \bar{\psi} \psi\right\}\right] .
\end{aligned}
$$

In our analysis we will mainly consider two scenarios:

(i) Universal coupling scenario $\left(\eta=\eta_{1}\right)$ : Both SM and DM can couple to the torsion field with a universal coupling $\eta$, which is a natural outcome of torsion portal. The model, thus naturally becomes very predictive as only a minimal set of input parameters are required to compute the DM phenomenology.

(ii) Non-universal coupling (NU) scenario $\left(\eta \neq \eta_{1}\right)$ : Though the universal coupling scenario is natural, but can be highly constrained in terms of the available parameter space. We shall illustrate this in detail in the subsequent sections. The situation may be considerably improved if one relaxes the universality condition, and set only the DM-torsion coupling $\eta$ as free parameter. We shall also briefly discuss the outcome of such a scenario where $\eta$ is different from torsion-SM coupling $\eta_{1}$.

\section{CONSTRAINTS ON THE MODEL PARAMETERS}

In this section we will discuss the constraints arising from various experimental and theoretical bounds. Under the universal coupling scenario (this is always be the case unless otherwise stated), our model can be characterized by only three free parameters, namely: 


$$
\left\{m_{s}, m_{\psi}, \eta\right\},
$$

which respectively are the torsion mass, the DM mass and the torsion-matter universal coupling. The constraints on the DM mass come from the requirement of obtaining observed relic abundance and satisfying the direct detection bounds. We discuss these in detail in the next section. Here we will mainly summarize the experimental constraints on the torsion mass and its coupling focusing on the earlier accelerator searches and recent LHC results.

(i) Before the advent of LHC, torsion mass and couplings were not entirely free parameters, rather significantly constrained from different analysis, including accelerator searches. Assuming the torsion mass to be considerably heavier than other particles present in the action [Eq. (10)], torsion interaction with fermion fields can be effectively recasted in terms of four-fermion contact interaction $[44,45]$.

$$
\mathcal{L}_{\text {int }}=-\frac{\eta^{2}}{m_{s}^{2}}\left(\bar{f} \gamma^{\mu} \gamma^{5} f\right)\left(\bar{f} \gamma^{\mu} \gamma^{5} f\right) .
$$

Now, such contact interactions can be constrained from several experiments as elaborated in $[44,45]$. From global analysis one can put a limit on the contact axial eeqq interaction, which then translates into a bound on the torsion mass and its coupling [43,44]:

$$
\frac{m_{s}}{\eta}>1.7 \mathrm{TeV}
$$

For a light torsion with mass below $1 \mathrm{TeV}$, accelerator searches from LEP or TEVATRON collaborations became important. Analysis of the data constraints the coupling $\eta$ in the range $0.02 \lesssim \eta \lesssim$ 0.1 [44,45], depending upon torsion mass. A combined analysis from LEP-2 and TEVATRON data excludes torsion mass upto $600 \mathrm{GeV}$ unless the torsion-fermion coupling drops below 0.1. Also, for torsion coupling $(\sim 0.3-0.6)$, the torsion mass is pushed well above TeV scale.

In recent years both ATLAS and CMS collaborations have searched for high-mass resonances through their direct production and subsequent decays to dilepton, dijet, or mono- $X$ final states [52-61]. Dilepton final states are of special interests as they provide the most stringent bounds, though other searches namely dijet and monojet limits [53-56] may also be of importance [62]. Based on the dilepton searches, that includes the recent CMS analysis at $13 \mathrm{TeV}$ center of mass (CM) energy with integrated luminosity $140 \mathrm{fb}^{-1}$ [63], a bound on heavy resonances, e.g., $m_{s} \gtrsim 4.5 \mathrm{TeV}$ can be placed assuming fermion coupling to torsion is different than the fermion couplings with the $Z$ boson. In fact, a similar limit for $\eta \sim 0.1$ was earlier presented in [49] where authors analyzed LHC discovery reach in the $\eta-m_{s}$ plane at $14 \mathrm{TeV}$ $\mathrm{CM}$ energy with integrated luminosity of $100 \mathrm{fb}^{-1}$. However, they considered only SM fermions in their analysis. Torsion specific analysis has also been performed by the ATLAS collaboration at $\sqrt{s}=7 \mathrm{TeV}$, for the same $e^{-}$and $\mu^{-}$final states and the bound is much more relaxed compared to $[49,63]$. One may also refer to the latest ATLAS $t \bar{t}$ production results to set limits on torsion parameters considering torsion-top quark couplings $O(1)$ [51]. Here a much weaker constraint $m_{s} \gtrsim 3 \mathrm{TeV}$ can be observed.

In this analysis, our primary objective is to study the interplay of different input parameters in DM phenomenology considering universal coupling scenario in particular. So, on the very outset, we do not impose any collider constraint on the parameter space. After investigating the DM phenomenology, limits are placed to depict the net available parameter space. In applying the LHC bounds, one should keep in mind that such limits on the torsion mass have been derived assuming narrow width approximation (NWA) while studying the distribution of the invariant mass of the decay products. However, this approximation does not hold good in the limit $\Gamma_{s} / m_{s} \geq 0.5$ [36,64], where perturbative limits break down. In this regime direct application of LHC limits may not be completely justified. Keeping this in mind we indicate the part of the parameter space where indeed one may have $\Gamma_{s} / m_{s} \geq 0.5$.

(ii) The propagating axial vector field $S_{\mu}$ may also contribute to the muon anomalous magnetic moment $\left(a_{\mu}\right)$ at one loop, though the contribution is found to be negative: $a_{\mu}^{S}\left(\mathrm{~m}_{\mathrm{s}} \gg \mathrm{m}_{\mu}\right) \sim-\frac{\eta^{2}}{4 \pi^{2}} \frac{5 \mathrm{~m}_{\mu}^{2}}{3 \mathrm{~m}_{\mathrm{s}}^{2}}[47,65]$. From the recent measurements of $a_{\mu}$ [66] one may observe the discrepancy in $\Delta a_{\mu}$ is positive: $(268 \pm 76.27) \times$ $10^{-11}$ which is an excess of $\sim 3.51 \sigma$ compared to the SM expectation. Thus, only a positive contribution from the BSM physics can match the difference which is not favoured in the present case.

(iii) A theoretical constraint can arise from the requirement of the preservation of unitarity of the theory due to quantum corrections [44-46,50]. This demands:

$$
\frac{m_{s}^{2}}{\eta} \gg m_{f}^{2},
$$

where $m_{f}$ is the mass of any fermion field that couples to the torsion. This constraint, however, is satisfied for our entire parameter space.

\section{DARK MATTER THROUGH TORSION PORTAL: WIMP SCENARIO}

As explained earlier, we consider the DM phenomenology of a singlet Dirac fermion $\psi$ in presence of a torsion 

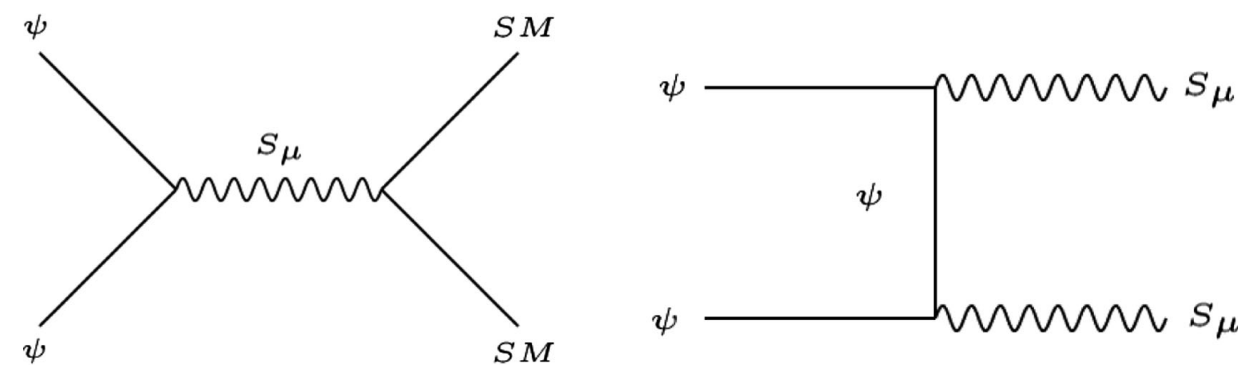

FIG. 1. Pair-annihilation of the DM $\psi$ to the SM particles via torsion-portal (left) and to the torsion pairs via $t$-channel process (right).

field where interactions between the SM fermions or the DM with the torsion is parametrized by a universal coupling parameter $\eta$. We remind that the DM is stable over the time scale of the age of the universe without requiring any ad hoc symmetry. This is also true in the parameter space where DM is heavier than the torsion field.

In the WIMP paradigm, the DM particles are assumed to be in thermal equilibrium with the SM in the early universe. In the present set-up, the thermal equilibrium is maintained through the torsion mediated $s$-channel and $t$-channel processes as shown in Fig. 1. At some later time when the rate of interaction becomes smaller than the Hubble rate of expansion the DM falls out of the equilibrium, and this defines freeze out $[1,2]$ of the DM:

$$
\Gamma_{A}<H
$$

where $\Gamma_{A}$ is the interaction rate of the DM pair annihilations and it is given by:

$$
\Gamma_{A}=n_{\psi}\langle\sigma v\rangle,
$$

where $n_{\psi}$ is the DM number density and $H$ is the Hubble constant. The present number density of the DM is decided by its number density at the time of freeze-out, which can be computed by using the standard prescription of Boltzmann equation [67] for a single component DM candidate. Note that, the relic abundance of the DM is solely controlled by the $2 \rightarrow 2$ annihilation channels, where the annihilation cross sections are dependent on the parameters in Eq. (11). In the present case, dominant nonrelativistic form of the thermally averaged annihilation cross section for the $s$ channel $(\psi \bar{\psi} \rightarrow \bar{f} f)$ and $t$ channel $\left(\psi \bar{\psi} \rightarrow S_{\mu} S_{\mu}\right.$ when kinematically allowed) processes can be expressed as:

$$
\begin{aligned}
\langle\sigma v\rangle_{\bar{f} f} \simeq & \sum_{f} N_{c}^{f} \frac{\eta^{4}}{2 \pi} \frac{m_{f}^{2}}{m_{s}^{4}} \sqrt{1-\frac{m_{f}^{2}}{m_{\psi}^{2}}}\left(\frac{\left(m_{s}^{2}-4 m_{\psi}^{2}\right)^{2}}{\left(m_{s}^{2}-4 m_{\psi}^{2}\right)^{2}+\Gamma_{s}^{2} m_{s}^{2}}\right) \\
& +\mathcal{O}\left(v^{2}\right), \\
\langle\sigma v\rangle_{s s} \simeq & \frac{\eta^{4}}{16 \pi m_{\psi}^{2}}\left(1-\frac{m_{s}^{2}}{m_{\psi}^{2}}\right)^{3 / 2}\left(1-\frac{m_{s}^{2}}{2 m_{\psi}^{2}}\right)^{-2}+\mathcal{O}\left(v^{2}\right),
\end{aligned}
$$

where $v$ is the relative velocity of the annihilating DM pair, $m_{f}$ corresponds to the mass of all SM fermions that couple to the torsion and $N_{c}^{f}$ refers the number of colors for final state fermions (=3(1) for quarks (leptons)). $\Gamma_{s}$ is the total decay width of the torsion to all fermions given by (in the rest frame of the torsion):

$\Gamma_{s}=\frac{N_{c}^{f} \eta^{2} m_{s}}{12 \pi}\left[1-4\left(\frac{m_{f}}{m_{s}}\right)^{2}\right]^{\frac{3}{2}}+\eta^{2} \frac{m_{s}}{12 \pi}\left[1-4\left(\frac{m_{\psi}}{m_{s}}\right)^{2}\right]^{\frac{3}{2}}$,

where the last term is relevant for $m_{s}>2 m_{\psi}$. The cross section has been computed for $s=4 m_{\psi}^{2}$ ( $s$ is the CM energy). $\mathcal{O}\left(v^{2}\right)$ refers to the velocity dependent $p$-wave terms, which are subdominant in the presence of a $s$-wave. It is intriguing to note that for the $s$-channel process, assuming $m_{\psi} \sim m_{s} \gg m_{f}$, the dominant $v^{2}$ contribution involves $\frac{\eta^{4} m_{\psi}^{2}}{\left(4 m_{\psi}^{2}-m_{s}^{2}\right)^{2}}$, which carries the resonance effect $[68,69]$. Interestingly the leading order term in Eq. (17) may not be sensitive to Breit-Wigner type narrow width resonance. The $p$-wave terms can also lead to important contributions for the relic density calculations, specially when the DM is lighter than top quark. Then the contribution of the leading order i.e., the velocity independent term in the $s$ channel [see Eq. (17)] is extremely suppressed $\sim \frac{m_{f}^{2}}{m_{s}^{4}}$, where $f$ refers any fermions except the top quark. The $p$-wave term becomes significant as it goes as $\sim \frac{1}{m_{\psi}^{2}}$ for $m_{\psi} \sim m_{s}$. Similarly $t$ channel contributions, free from $v^{2}$ suppression, are also important for $m_{s} \lesssim m_{\psi}$. For numerical calculations, we use the general expressions for $\langle\sigma v\rangle$. This has been done by implementing the model in CalcHEP [70] and then the model output has been fed to public code MicrOmegas [71-74]. The valid parameter space should comply with the observed relic abundance data $[75,76]$ :

$$
\Omega_{\psi}^{\mathrm{obs}} h^{2}=0.1199 \pm 0.0022
$$

For illustration we first fixed the DM mass $m_{\psi}=$ $500 \mathrm{GeV}$ and plotted the variation of relic abundance with $m_{s}$ for four different choices of the $\eta:\{0.1,0.3,0.5,0.7\}$ 

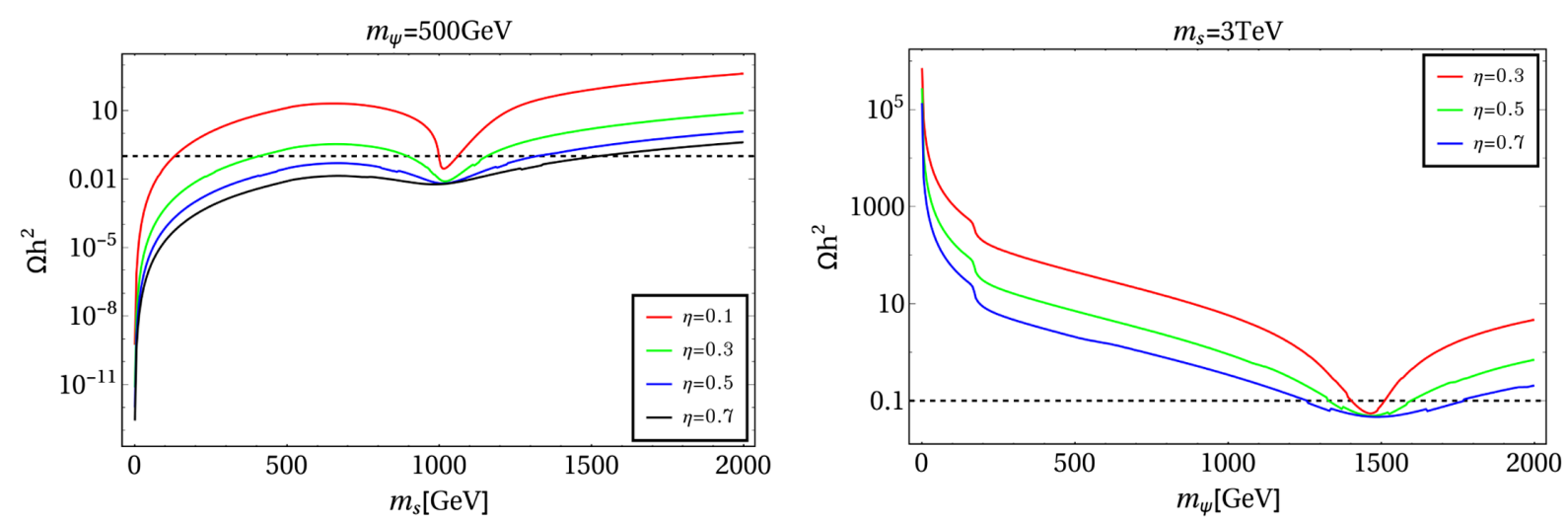

FIG. 2. Left: Variation of relic abundance of $\psi$ with $m_{s}$ for different choices of the couplings $\eta:\{0.1,0.3,0.5,0.7\}$, are shown in red, green, blue, and black respectively. Here the DM mass is set at $500 \mathrm{GeV}$. Right: Same for a fixed torsion mass $m_{s}=3 \mathrm{TeV}$ for three different choices of $\eta:\{0.3,0.5,0.7\}$ respectively in red, green, and blue. In each figure the black dashed line corresponds to the PLANCK observed relic abundance.

respectively which are shown by red, green, blue and black curves on the left-hand side (LHS) of Fig. 2. As expected, in each case we see a resonance at $m_{\psi} \simeq \frac{m_{s}}{2}$ where the observed relic abundance [76] is satisfied. The relic abundance increases for smaller $\eta$, and at resonance it is difficult to produce an acceptable $\Omega h^{2}$ for $\eta<0.1$. The resonance regions may become flat for a larger $\eta$ when the Breit-Wigner decay width $\Gamma_{s}$ is large for a given torsion mass $m_{s}$. The situation is different for a lighter $S_{\mu}$ hence for a lighter DM, as both $s$ and $t$ channel processes may become relevant here. As an example, for our chosen DM mass, $\langle\sigma v\rangle_{\bar{\psi} \psi \rightarrow \bar{t} t}$ can be sufficiently large even for a moderate value of $\eta$, where right relic abundance or even a under abundant region can be observed. Here annihilations are facilitated to all possible final states. The viable parameter space may also include the region where $m_{s} \lesssim m_{\psi}$. Next, we fix the torsion mass $m_{s}=3 \mathrm{TeV}$ and choose three different couplings $\eta:\{0.3,0.5,0.7\}$ as shown on the right-hand side (RHS) of Fig. 2 through red, green, and blue curves respectively. Here the relic density is satisfied only at the resonance region while larger width can be observed for a larger $\eta$.

Now, in order to explore the parameter space satisfying the observed relic abundance [via Eq. (20)] we scan over the parameters listed in Eq. (11) in the following range:

$m_{\psi}:\{10-5000 \mathrm{GeV}\} ; m_{s}:\left\{10-10^{4} \mathrm{GeV}\right\} ; \eta:\{0.01-1.0\}$.

The corresponding parameter space is shown in Fig. 3. On the LHS we show the parameter space satisfying relic abundance in $m_{\psi}-\eta$ plane for different ranges of the torsion mass $m_{s}$ indicated by different colours. Here both DM and the torsion can take small mass values $\sim 10 \mathrm{GeV}$ in different parts of the parameter space specified by $\eta$, where relic abundance can be satisfied either through annihilations to $f \bar{f}$ or via $t$ channel process.

In the RHS of Fig. 3, relic density allowed region is shown in $m_{\psi}-m_{s}$ plane where different colours correspond to different values of the coupling $\eta$. We see here,

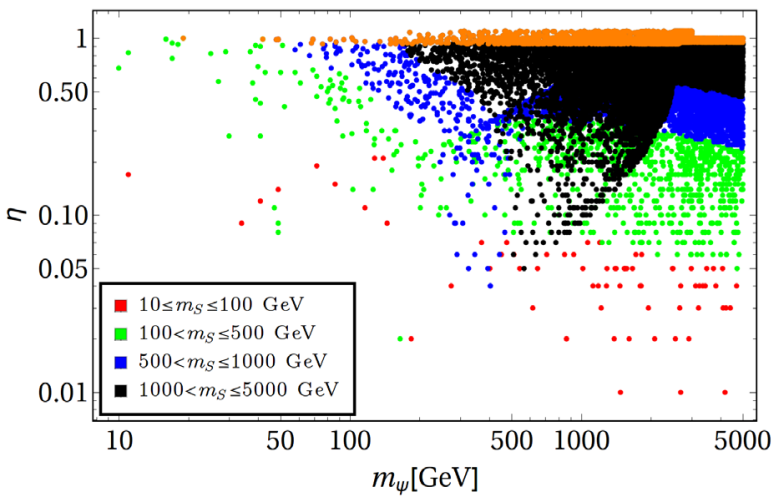



FIG. 3. Left: Relic density satisfied parameter space in $m_{\psi}-\eta$ plane, where different colors show torsion masses in the range $\{10-100\} \mathrm{GeV}$ in red, $\{100-500\} \mathrm{GeV}$ in green, $\{500-1000\} \mathrm{GeV}$ in blue, and $\{1000-5000\}$ GeV in black. Right: Parameter space satisfying relic abundance in $m_{\psi}-m_{s}$ plane, where the color codes corresponding to different values of the coupling $\eta:\{0.01-0.05\}$ in red, $\{0.05-0.1\}$ in green, $\{0.1-0.5\}$ in blue, and $\{0.5-1.0\}$ in black. In each plot the orange region corresponds to $\Gamma_{s} / m_{s} \geq 0.5$ limit. 
smaller values of $\eta$ (in red) favours $m_{s} \lesssim 100 \mathrm{GeV}$, while a larger $m_{s}$ is required for larger $\eta$ (in blue and black) to satisfy the relic abundance. This is expected as the rise in the torsion mass reduces $\langle\sigma v\rangle$. Hence larger $\eta$ is expected to make relic density compatible with the observed abundance. In each plot highlighted orange regions depict $\Gamma_{s} / m_{s} \geq 0.5$, where LHC constraints may not be directly applied to disqualify a parameter space point as discussed in Sec. III.

\section{A. Indirect detection of the dark matter}

The relic density allowed parameter space for the DM can also be probed via different indirect detection experiments that look for annihilation and/or decay products of the DM that produces SM particles. Residual DM annihiliations may still occur at late universe where the relative velocity takes a value $\sim 10^{-3}$. Among these final states, photon and neutrinos, being neutral and stable, can reach the detectors without getting much affected by the intermediate space. In case of high energy $\gamma$-rays, one considers hadronization and production of neutral pions and its subsequent decays to two photons with varying energies and also final state radiations. Detectable gamma-rays can be tested at the instruments like the Fermi Large Area Telescope (LAT) ${ }^{4}$ in space or the ground-based Cherenkov telescopes like MAGIC. ${ }^{5}$ Since no signal has so far been observed, we would like to see the status of the relic abundance satisfying parameter space from the bounds arising from a joint global analysis from the MAGIC and the Fermi-LAT which search for gamma-ray signals from DM annihilation in dwarf spheroidal galaxies (dSphs) [77].

The differential gamma ray flux produced due to WIMPlike DM annihilation in a given region of the sky $(\Delta \Omega)$ and observed at the Earth reads [78-82]:

$$
\frac{d \Phi}{d E}(\Delta \Omega)=\frac{1}{4 \pi} \frac{\langle\sigma v\rangle J(\Delta \Omega)}{2 m_{\psi}^{2}} \frac{d N}{d E},
$$

where $d N / d E$ is the average gamma-ray spectrum per annihilation and,

$$
J(\Delta \Omega)=\int_{\Delta \Omega} d \Omega^{\prime} \int_{\operatorname{LOS}} d l \rho^{2}\left(l, \Omega^{\prime}\right),
$$

is the well-known $J$-factor with $\rho$ being the DM density profile, and the integrals running over $\Delta \Omega$ and the line-ofsight (LOS) through the DM distribution. Here we consider a Navarro-Frenk-While (NFW) profile for the computation [83]. Since the DM $\psi$ can annihilate into charged fermion final states: $\mu^{+} \mu^{-}, \tau^{+} \tau^{-}, b \bar{b}$ etc, all such final states can give rise to gamma rays. Thus, one can constrain the DM annihilations to different charged final states from non

\footnotetext{
${ }_{5}^{4}$ http://fermi.gsfc.nasa.gov.

${ }^{5}$ https://magic.mpp.mpg.de/.
}

observations of gamma rays. In Fig. 4 we bounded our relic density allowed parameter space from MAGIC and FermiLAT data [77] for muon (top left), tau (top right) and $b$-quark (bottom) final states. We see that for our choice of the coupling $\eta$ and torsion mass $m_{s}$, the parameter space is completely unconstrained. This is not completely unexpected, as the velocity dependent contributions in Eq. (17) which may show the resonance behavior is largely suppressed at late times.

\section{B. Direct detection of the dark matter}

Since the DM can annihilate into the SM particles via $s$-channel mediation of the torsion producing right relic abundance, the same interaction can give rise to DMnucleon elastic scattering. This will lead to the direct detection of the DM in present and future experiments. In the present case, driven by a purely axial coupling, the DM-nucleon scattering would give rise to only spindependent interaction. Note that, the SD cross section involves the spin content of the nucleus i.e., there is a $J(J+1)$ enhancement from the nuclear spin $J$. This is highly welcome since a weak-scale Dirac dark matter accompanied by vectorlike interactions with the some mediator $M_{\mu}$, i.e., $\left(\psi \gamma^{\mu} \psi\right) M_{\mu}$ is either ruled out or very tightly constrained. The relevant Feynman graph for the spin-dependent scattering is depicted in Fig. 5. For such a process, the spin-dependent direct search cross section is proportional to [72]:

$$
\sigma_{\mathrm{SD}} \sim \frac{\eta^{4}}{m_{s}^{4}} \frac{16}{\pi} \mu^{2}\left(\frac{J+1}{J}\right)\left(\xi_{p}\left\langle s_{p}\right\rangle+\xi_{n}\left\langle s_{n}\right\rangle\right)^{2},
$$

where $\mu=\frac{m_{\psi} m_{N}}{m_{\psi}+m_{N}}$ is the DM-nucleon reduced mass with $N \in\{n, p\}$, i.e., neutron or proton. $\left\langle s_{p, n}\right\rangle$ are obtained from nuclear calculations or from simple nuclear models, such as the odd-group model [72] and they are estimated to be $\approx 0.5$ for a nuclei with an odd number of protons or neutrons, while zero for nuclei with even number of protons or neutrons. $\xi_{p, n}$ are obtained from lattice data $[72,84,85]$. We consider the most recent limit on spin-dependent WIMP-proton or WIMP-neutron cross section from the run-3 data of the LUX experiment [86] and constrain our relic density satisfying parameter space. In both the plots $\mathrm{DM}$ and torsion mass span over a few $\mathrm{TeV}$ mass scale and $\eta:\{0.01-1.0\}$. From the top panel in Fig. 6 we see that the relic density allowed parameter space lies well below the present bound from LUX. This is due to the additional suppression coming from the torsion mass in Eq. (24). Similarly, smaller coupling results in even smaller cross section. In the plots, we also show projected sensitivity of the next generation LUX-ZEPLIN (LZ) direct detection experiments which may in future probe some parts of the parameter space [87]. Clearly, SD neutron-DM scattering offers a better prospect which can be attributed to even 

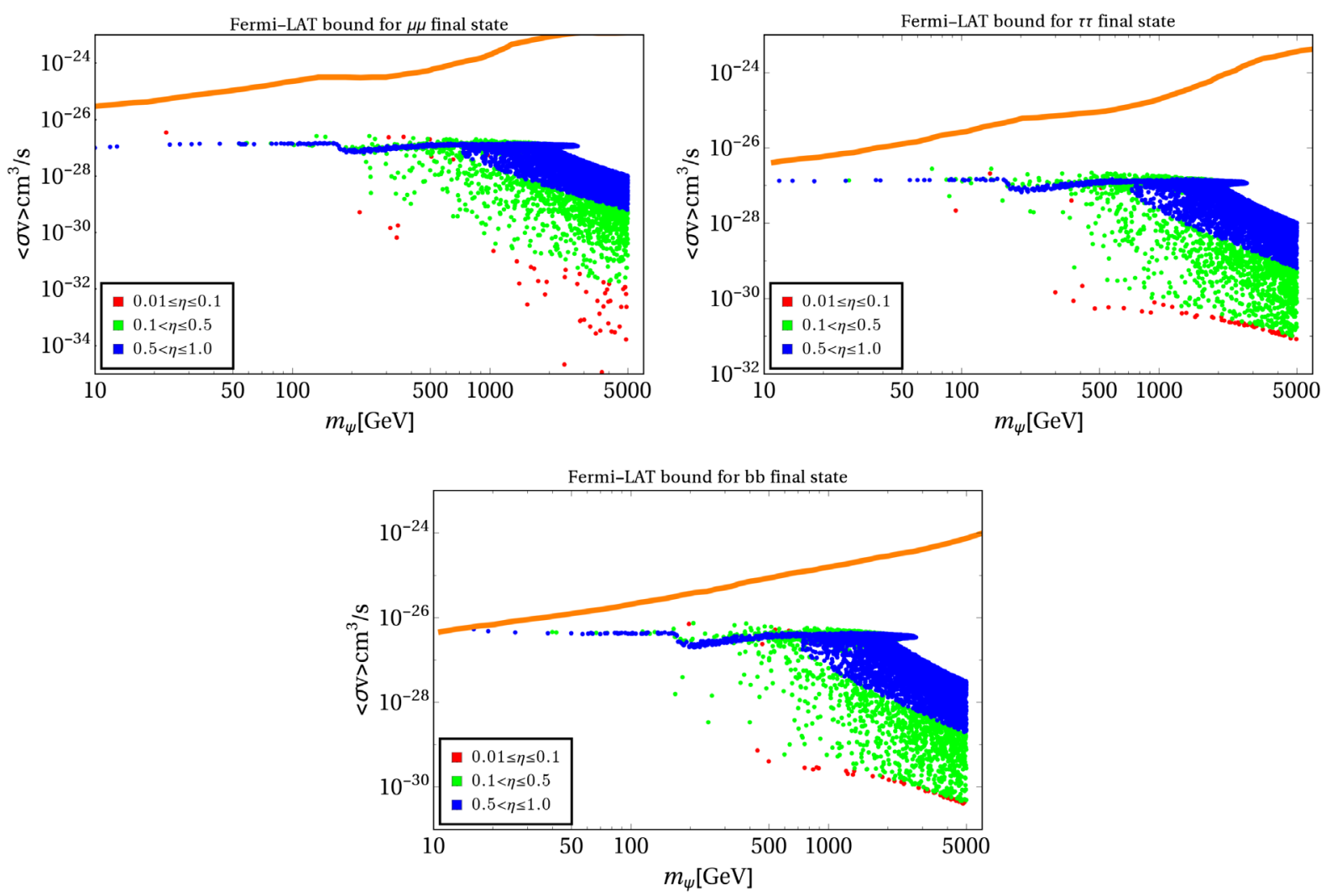

FIG. 4. DM annihilations into $\mu^{+} \mu^{-}$(top left), $\tau^{+} \tau^{-}$(top right) and $b \bar{b}$ (bottom) compared against indirect detection bounds from the combined observation of MAGIC and Fermi-LAT. In each figure regions corresponding different couplings are shown in red $\{0.01-0.1\}$, green $\{0.1-0.5\}$, blue $\{0.5-1.0\}$. The thick orange curve represents bound from nonobservations of $\gamma$-rays corresponding to particular final state.

number of protons present in the xenon-based detectors like LUX [88]. Finally in the bottom left panel of Fig. 6 we show the net parameter space satisfying relic abundance and direct search exclusion limit. We find that if we strictly follow $\Gamma_{s} / m_{s} \lesssim 0.5$, the parameter space has shrunk a bit in the high torsion mass region with $\eta \lesssim 0.8$.



FIG. 5. DM-nucleon elastic scattering mediated by the torsion.

\section{LIMITS ON THE UNIVERSAL COUPLING SCENARIO AND NONUNIVERSAL TORSION MATTER COUPLING}

Based on our discussion in Sec. III, it is quite expected that our minimal coupling scenario would be tightly bounded from the direct production of torsion and its subsequent decays to charged leptons: $e, \mu$. For a qualitative understanding, we calculated effective cross section for dilepton productions $\sigma\left(p p \rightarrow S_{\mu}\right) \times \operatorname{Br}\left(S_{\mu} \rightarrow l^{+} l^{-}\right)$ $(l \in e, \mu)$ and plotted the variation with $m_{s}$ for different sets of torsion-matter couplings. Torsion production cross section $\sigma\left(p p \rightarrow S_{\mu}\right)$ at the LHC has been calculated using MadGraph-v2.6.6 [89] at a CM energy of $13 \mathrm{TeV}$ with PDF choice CTEQ6L [89-93] while the branching fraction has been determined from Eq. (19). The result is presented in Fig. 7, where the exclusion limit is from [58]. The bound would be relaxed if one considers finite width of the mediator [59]. Torsion mass in the range $m_{s} \sim\{3-5\} \mathrm{TeV}$ is typically allowed modulo the universal coupling $\eta$ is small $\eta:\{0.01-0.1\}$. However, in this range, satisfying the PLANCK data will be extremely difficult as one requires relatively light $S_{\mu}$ to compensate the coupling suppression in order to produce relic abundance in the right ballpark. In other words, the typical $m_{s}$ value which can satisfy the 

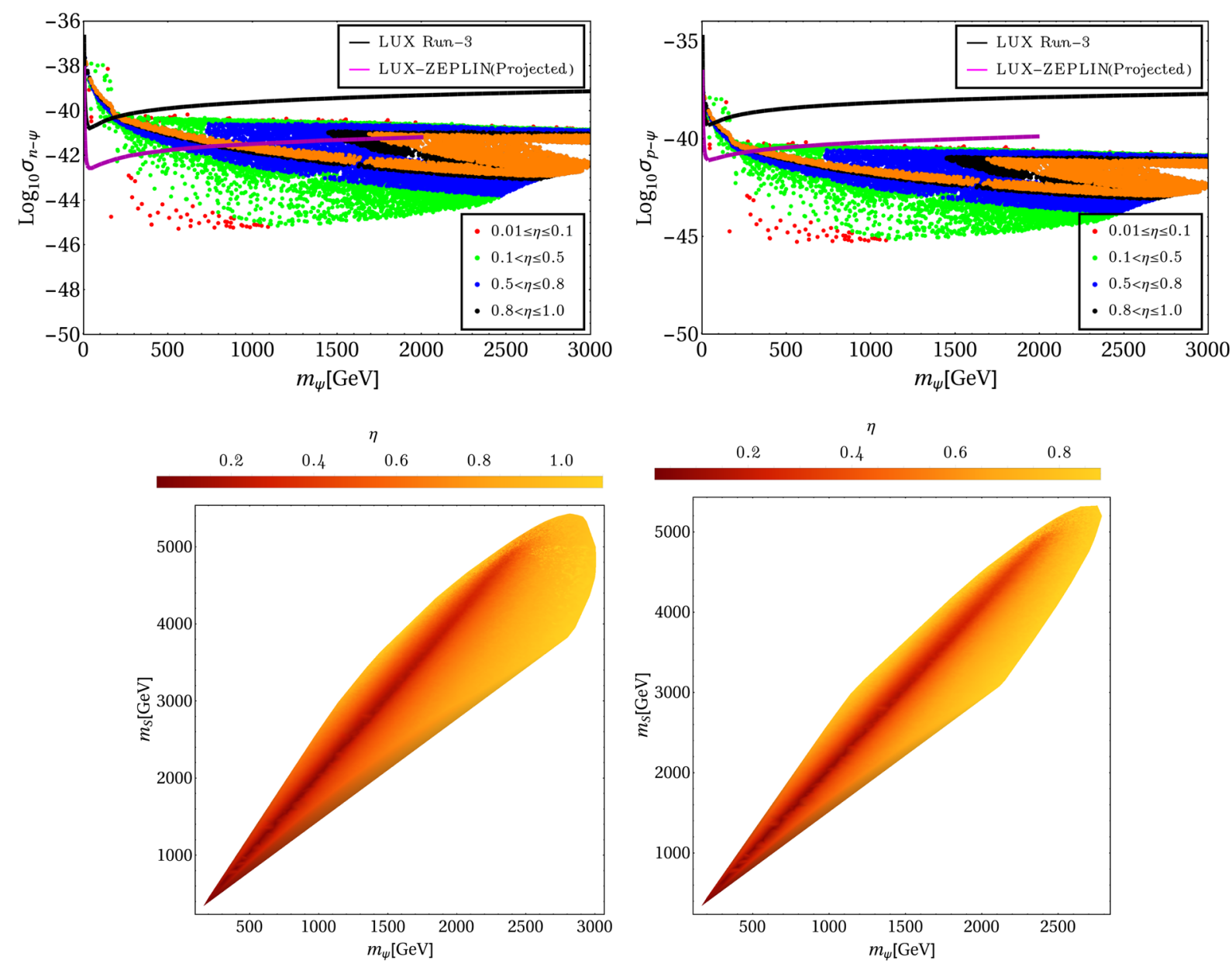

FIG. 6. Top left: Relic density satisfied parameter space in direct search plane, where different colours correspond to different choices of $\eta:\{0.01-0.1\}$ in red, $\{0.1-0.5\}$ in green, $\{0.5-0.8\}$ in blue, and $\{0.8-1.0\}$ in black. The black dashed line shows the exclusion limit from spin-dependent direct search for neutron-DM scattering. Similarly, the magenta line refers future LZ sensitivity. Top right: Same but here the exclusion limits refer spin-dependent direct search for proton-DM scattering. In both plots orange region corresponds to $\Gamma_{s} / m_{s} \geq 0.5$. Bottom left: Net parameter space satisfying relic abundance and spin-dependent direct search in $m_{\psi}-m_{s}$ plane, where the top panel shows the variation of the coupling $\eta$. Bottom right: Net parameter space within the perturbative limit $\Gamma_{s} / m_{s} \lesssim 0.5$.



FIG. 7. $\sigma\left(p p \rightarrow S_{\mu}\right) \times \operatorname{Br}\left(S_{\mu} \rightarrow l^{+} l^{-}\right)(l \in e, \mu)$ vs. $m_{s}$ in the universal coupling scenario. observed abundance for a given $\eta$ in the aforesaid range has been mostly ruled out by the LHC results. On the higher side of the coupling $\eta \sim 1$, one mostly finds $\frac{\Gamma}{m_{s}}>0.5$, thus LHC results cannot be used to rule out any parameter space point.

The situation can be improved a lot when one assumes a different value of the coupling parameter for DM-torsion $(\eta)$ relative to the SM fermions-torsion which is parametrized by $\eta_{1}$, as introduced in Eq. (10). As discussed, we refer this scenario as nonuniversal torsion matter coupling (NU) model. In this case, we always keep $\eta_{1} \sim 0.01$ which allows $m_{s}>3 \mathrm{TeV}$ to comply with the LHC dilepton results, while $\eta$ has been varied within $\{0.1-1\}$. With this choice of torsion-matter couplings, $\operatorname{Br}\left(S_{\mu} \rightarrow l^{+} l^{-}\right)$would be further reduced, thanks to rise in invisible branching fraction of the torsion. In the left side of Fig. 8 we place bounds on $m_{s}$ for different choices of $\eta=0.05$, 

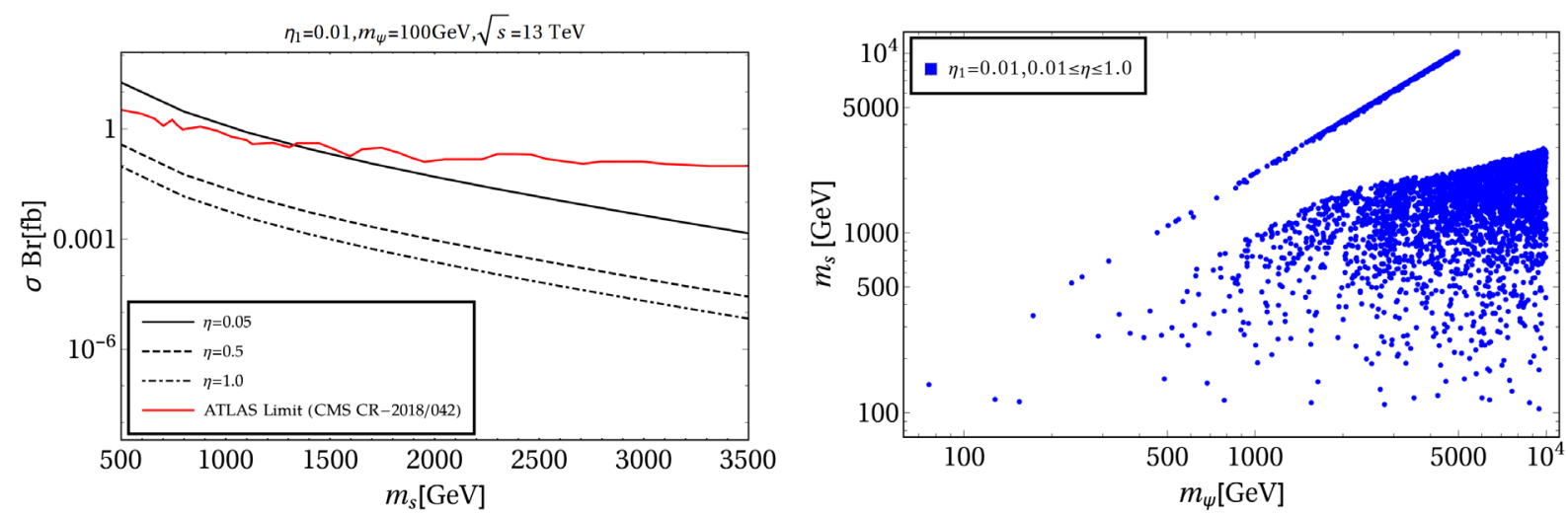

FIG. 8. Left: $\sigma\left(p p \rightarrow S_{\mu}\right) \times \operatorname{Br}\left(S_{\mu} \rightarrow l^{+} l^{-}\right)(l \in e, \mu)$ vs. $m_{s}$ in the nonuniversal coupling scenario. Here torsion-SM fermion couplings are set at $\eta_{1}=0.01$. In the minimal coupling case, the bound on $m_{s}$ reads as $m_{s}>3 \mathrm{TeV}$. But with a different torsion-DM coupling, torsion's branching fraction to leptonic final states will be reduced further to allow even lighter $S_{\mu}$. Assuming $\eta=0.05$, one finds $m_{s}>1.4 \mathrm{TeV}$ using LHC dilepton searches. Right: Allowed parameter space complied with PLANCK data in $m_{s}-m_{\psi}$ plane in the NU coupling scenario.

$0.5,1$ keeping $\eta_{1}=0.01$. For example for $\eta=0.05$, the bounds on $m_{s}$ reduced to $m_{s}>1.4 \mathrm{TeV}$ from dilepton searches. Further rise in $\eta$ would make torsion mass even less constrained due to increase in the invisible branching fractions. Similar to dilepton searches, dijet bounds would not be of much significance. The relevant limits may come from the monojet searches which is induced by the axial vector couplings [54,55]. According to an analysis in [54], which corresponds to $13 \mathrm{TeV} \mathrm{CM}$ energy and $12.9 \mathrm{fb}^{-1}$ integrated luminosity, torsion mass up to $\sim 2 \mathrm{TeV}$ has been ruled out if one assumes a coupling strength of 0.25 between the mediators and the SM fermions while a coupling strength of 1.0 between the mediators and the DM particles. Similar limit $m_{s}>1.55 \mathrm{TeV}$ can be set corresponding to an integrated luminosity of $36.1 \mathrm{fb}^{-1}$ [55]. For our chosen input parameters $\eta_{1}$ and $\eta$, the limits should get much less stringent due to strong suppression in the production cross section for $S_{\mu}$. In the RHS of Fig. 9, we depict the allowed parameter points by PLANCK data. Acceptable relic abundance can be obtained along two regions: (i) resonance annihilations, which pass almost through the middle of the plane and (ii) $s$ and $t$-channel processes when the torsion is lighter than the DM. A detailed study of monojet searches on the parameter space may further constrain the parameter space. However, this is beyond the scope of this paper.

Finally, we also explore the parameter space via the SD direct detections in the plane of neutron-DM and proton-DM plane. Like our previous results in the universal coupling scenario, unpaired neutrons in Xenon based detectors would help to make neutron-DM scattering more effective to constrain the parameter space. Part of the parameter space, specially the resonance annihilation regions, can be probed in the near future via LZ experiments.

\section{CONCLUSIONS}

In this paper we have explored the possibility of having a fermionic dark matter (DM) in an extension of the SM, where torsion field $\left(S_{\mu}\right)$ plays the role of portal. Guided by its geometric origin, the torsion field can naturally be realized as a massive axial vector field. The dark sector is
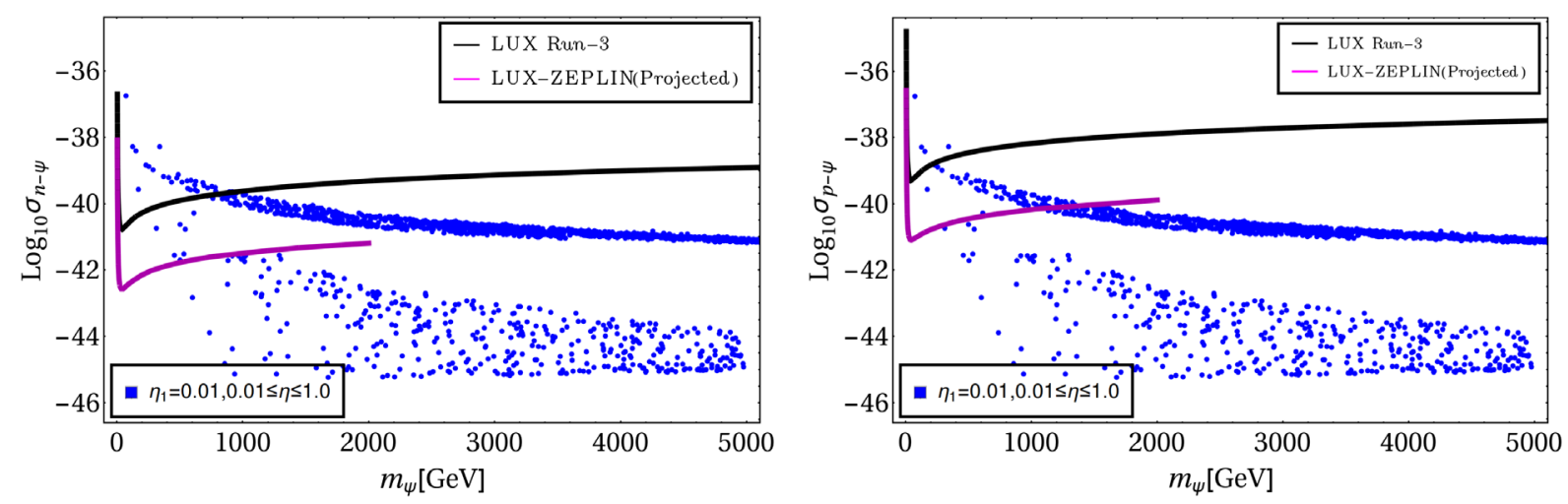

FIG. 9. Left:DM-neutron SD scattering that satisfies PLANCK data on relic abundance where limits from LUX and proposed LUX-ZEPLIN have been shown. Right: Same but for DM-proton SD scattering. 
comprised only of the SM-singlet Dirac fermion $(\psi)$ that makes up all of the observed DM relic abundance. The DM is naturally stable over the cosmological time scale (i.e., nondecaying) without the imposition of any ad hoc symmetry or kinematical condition to forbid its decay (in other words, the DM need not to be the lightest stable particle (LSP) in this setup). Thus the proposed model is a minimal extension of the SM. We consider two scenarios to parametrize the torsion-matter (includes both DM and SM particles) couplings. In the first case both the torsion-DM and torsion-SM couplings are assumed to be equal (dubbed as universal coupling scenario) at the low energy scale. It leads to a simplified DM phenomenology involving only three free parameters: the universal torsion-DM coupling $\eta$, DM mass $m_{\psi}$ and the torsion mass $m_{s}$. We consider the DM to be WIMP-like, and via a detailed parameter space scan we find that correct relic abundance can be achieved over a large parameter space for DM mass $m_{\psi}:\{10-5000\} \mathrm{GeV}$, with torsion mass lying in the same range and for $\eta \sim\{0.1-1\}$. The DM phenomenology is somewhat equivalent to popular $Z^{\prime}$-mediated DM models but unlike $Z^{\prime}$, the torsion can only accommodate axial couplings. However, a significant difference lies in the fact that in this setup the dark sector and the visible sector fermions can naturally enjoy the same coupling with the mediator. Parameter space of such a simple model is highly constrained from the recent LHC data. Thus, stringent bounds can be derived from the dilepton searches at the LHC similar to massive (axial) $Z^{\prime}$ searches. From our computation of the observable $\sigma\left(p p \rightarrow S_{\mu}\right) \times \operatorname{Br}\left(S_{\mu} \rightarrow l^{+} l^{-}\right)(l \in e, \mu)$, we find that $\eta \lesssim$ $0.01(0.1)$ is required for $m_{s} \lesssim 3(5) \mathrm{TeV}$ which places very tight constraint on the viable DM parameter space. In fact, the whole parameter space is mostly excluded except for $\eta \sim 1$, where large decay width of the torsion may invalidate the LHC limits. Then we move to the nonuniversal coupling scenario where torsion-DM interaction strength is assumed to be different from that of torsion-SM fermion (dubbed as nonuniversal coupling). Since LHC constraints are mainly determined by torsion-quark couplings, we set them at 0.01 and vary the DM-torsion couplings in the range $\{0.1-1\}$. Here $\operatorname{Br}\left(S_{\mu} \rightarrow l^{+} l^{-}\right)$is reduced due to rise in the invisible decay of the torsion, which essentially helps to have even lighter $S_{\mu}<3 \mathrm{TeV}$ allowed compared to the universal scenario. The PLANCK-observed relic abundance is satisfied along the resonance region and also for $m_{s} \leq m_{\psi}$. In both universal and nonuniversal scenarios direct detection of the DM has been computed. Here, the presence of the axial-vector interaction turns out to be beneficial as this gives rise only to a spin-dependent direct detection cross section. We find that almost all of the relic density allowed parameter space lies comfortably below the present exclusion limit of direct search experiments. However, future spin-dependent direct search experiments, e.g., LUX-ZEPLIN with higher sensitivity can probe some parts of the parameter space.

\section{ACKNOWLEDGMENTS}

The computation was supported in part by the SAMKHYA: High Performance Computing Facility provided by Institute of Physics, Bhubaneswar. T. B. acknowledges the local hospitality at IOP, Bhubaneswar where this work was started. D. D. acknowledges discussion with Sujoy Poddar.
[1] E. W. Kolb and M. S. Turner, Front. Phys. 69, 1 (1990).

[2] G. Jungman, M. Kamionkowski, and K. Griest, Phys. Rep. 267, 195 (1996).

[3] G. Bertone, D. Hooper, and J. Silk, Phys. Rep. 405, 279 (2005).

[4] J. L. Feng, Annu. Rev. Astron. Astrophys. 48, 495 (2010).

[5] G. Bertone and D. Hooper, Rev. Mod. Phys. 90, 045002 (2018).

[6] T. Lin, Proc. Sci. 333 (2019) 009 [arXiv:1904.07915].

[7] E. Aprile et al. (XENON100 Collaboration), Phys. Rev. Lett. 109, 181301 (2012).

[8] D. S. Akerib et al. (LUX Collaboration), Phys. Rev. Lett. 118, 021303 (2017).

[9] X. Cui et al. (PandaX-II Collaboration), Phys. Rev. Lett. 119, 181302 (2017).

[10] E. Aprile et al. (XENON Collaboration), Phys. Rev. Lett. 121, 111302 (2018).
[11] G. Arcadi, M. Dutra, P. Ghosh, M. Lindner, Y. Mambrini, M. Pierre, S. Profumo, and F. S. Queiroz, Eur. Phys. J. C 78, 203 (2018).

[12] G. Arcadi, Y. Mambrini, and F. Richard, J. Cosmol. Astropart. Phys. 03 (2015) 018.

[13] K. Hamaguchi and K. Ishikawa, Phys. Rev. D 93, 055009 (2016).

[14] M. Escudero, A. Berlin, D. Hooper, and M.-X. Lin, J. Cosmol. Astropart. Phys. 12 (2016) 029.

[15] J. Kearney, N. Orlofsky, and A. Pierce, Phys. Rev. D 95, 035020 (2017).

[16] J. Ellis, A. Fowlie, L. Marzola, and M. Raidal, Phys. Rev. D 97, 115014 (2018).

[17] O. Lebedev, H. M. Lee, and Y. Mambrini, Phys. Lett. B 707, 570 (2012).

[18] A. Djouadi, O. Lebedev, Y. Mambrini, and J. Quevillon, Phys. Lett. B 709, 65 (2012). 
[19] C. Gross, O. Lebedev, and Y. Mambrini, J. High Energy Phys. 08 (2015) 158.

[20] J. A. Casas, D. G. Cerdeo, J. M. Moreno, and J. Quilis, J. High Energy Phys. 05 (2017) 036.

[21] M. Hoferichter, P. Klos, J. Menndez, and A. Schwenk, Phys. Rev. Lett. 119, 181803 (2017).

[22] E. Hardy, J. High Energy Phys. 06 (2018) 043.

[23] G. Arcadi, A. Djouadi, and M. Raidal, Phys. Rep. 842, 1 (2020).

[24] M. Drees and M. M. Nojiri, Phys. Rev. D 47, 376 (1993).

[25] P. Nath and R. L. Arnowitt, Phys. Rev. Lett. 70, 3696 (1993).

[26] H. Baer and M. Brhlik, Phys. Rev. D 57, 567 (1998).

[27] J. R. Ellis, K. A. Olive, Y. Santoso, and V. C. Spanos, Phys. Lett. B 565, 176 (2003).

[28] U. Chattopadhyay and D. Das, Phys. Rev. D 79, 035007 (2009).

[29] U. Chattopadhyay, D. Das, D. K. Ghosh, and M. Maity, Phys. Rev. D 82, 075013 (2010).

[30] D. Das, A. Goudelis, and Y. Mambrini, J. Cosmol. Astropart. Phys. 12 (2010) 018.

[31] Y. Mambrini, J. Cosmol. Astropart. Phys. 09 (2010) 022.

[32] E. Dudas, L. Heurtier, Y. Mambrini, and B. Zaldivar, J. High Energy Phys. 11 (2013) 083.

[33] A. Alves, S. Profumo, and F. S. Queiroz, J. High Energy Phys. 04 (2014) 063.

[34] O. Lebedev and Y. Mambrini, Phys. Lett. B 734, 350 (2014).

[35] D. Hooper, Phys. Rev. D 91, 035025 (2015).

[36] A. Alves, A. Berlin, S. Profumo, and F. S. Queiroz, Phys. Rev. D 92, 083004 (2015).

[37] A. Alves, A. Berlin, S. Profumo, and F. S. Queiroz, J. High Energy Phys. 10 (2015) 076.

[38] B. Allanach, F. S. Queiroz, A. Strumia, and S. Sun, Phys. Rev. D 93, 055045 (2016); 95, 119902(E) (2017).

[39] A. Alves, G. Arcadi, Y. Mambrini, S. Profumo, and F. S. Queiroz, J. High Energy Phys. 04 (2017) 164.

[40] A. Berlin, S. Gori, T. Lin, and L.-T. Wang, Phys. Rev. D 92, 015005 (2015).

[41] S. Baek, P. Ko, and J. Li, Phys. Rev. D 95, 075011 (2017).

[42] M. Bauer, M. Klassen, and V. Tenorth, J. High Energy Phys. 07 (2018) 107.

[43] I. L. Shapiro, Phys. Rep. 357, 113 (2002).

[44] A. S. Belyaev and I. L. Shapiro, Phys. Lett. B 425, 246 (1998).

[45] A. S. Belyaev and I. L. Shapiro, Nucl. Phys. B543, 20 (1999).

[46] G. de Berredo-Peixoto, J. A. Helayel-Neto, and I. L. Shapiro, J. High Energy Phys. 02 (2000) 003.

[47] P. Das, U. Mahanta, and S. Raychaudhuri, arXiv:hep-ph/ 0211137.

[48] U. Mahanta and S. Raychaudhuri, arXiv:hep-ph/0307350.

[49] A. S. Belyaev, I. L. Shapiro, and M. A. B. do Vale, Phys. Rev. D 75, 034014 (2007).

[50] A. S. Belyaev, M. C. Thomas, and I. L. Shapiro, Phys. Rev. D 95, 095033 (2017).

[51] F. M. L. De Almeida, F. R. De Andrade, M. A. B. do Vale, and A. A. Nepomuceno, Phys. Rev. D 97, 075036 (2018).

[52] G. Aad et al. (ATLAS Collaboration), J. High Energy Phys. 11 (2012) 138.
[53] A. M. Sirunyan et al. (CMS Collaboration), Phys. Lett. B 769, 520 (2017); 772, 882(E) (2017).

[54] A. M. Sirunyan et al. (CMS Collaboration), J. High Energy Phys. 07 (2017) 014.

[55] M. Aaboud et al. (ATLAS Collaboration), J. High Energy Phys. 01 (2018) 126.

[56] M. Aaboud et al. (ATLAS Collaboration), Phys. Rev. D 96, 052004 (2017).

[57] A. M. Sirunyan et al. (CMS Collaboration), J. High Energy Phys. 06 (2018) 120.

[58] R. Goldouzian (ATLAS, CMS and SUSY Collaborations), Tech. Rep. CMS-CR-2018-042, CERN, Geneva, 2018.

[59] A. White (ATLAS Collaboration), in Proceedings of the 54th Rencontres de Moriond on Gravitation (Moriond Gravitation 2019) La Thuile, Italy, 2019 (2019).

[60] G. Aad et al. (ATLAS Collaboration), Phys. Lett. B 796, 68 (2019).

[61] A. M. Sirunyan et al. (CMS Collaboration), Phys. Lett. B 798, 134952 (2019).

[62] M. Drees and Z. Zhang, Phys. Lett. B 797, 134832 (2019).

[63] CMS Collaboration, Tech. Rep. CMS-PAS-EXO-19-019, CERN, Geneva, 2019.

[64] J. Abdallah et al., arXiv:1409.2893.

[65] F. S. Queiroz and W. Shepherd, Phys. Rev. D 89, 095024 (2014).

[66] M. Tanabashi and E. A. Hagiwara (Particle Data Group Collaboration), Phys. Rev. D 98, 030001 (2018).

[67] P. Gondolo and G. Gelmini, Nucl. Phys. B360, 145 (1991).

[68] A. Berlin, D. Hooper, and S. D. McDermott, Phys. Rev. D 89, 115022 (2014).

[69] G. Arcadi, M. D. Campos, M. Lindner, A. Masiero, and F. S. Queiroz, Phys. Rev. D 97, 043009 (2018).

[70] A. Belyaev, N. D. Christensen, and A. Pukhov, Comput. Phys. Commun. 184, 1729 (2013).

[71] G. Belanger, F. Boudjema, A. Pukhov, and A. Semenov, Comput. Phys. Commun. 176, 367 (2007).

[72] G. Belanger, F. Boudjema, A. Pukhov, and A. Semenov, Comput. Phys. Commun. 180, 747 (2009).

[73] G. Belanger, F. Boudjema, A. Pukhov, and A. Semenov, Comput. Phys. Commun. 185, 960 (2014).

[74] D. Barducci, G. Belanger, J. Bernon, F. Boudjema, J. Da Silva, S. Kraml, U. Laa, and A. Pukhov, Comput. Phys. Commun. 222, 327 (2018).

[75] N. Jarosik et al., Astrophys. J. Suppl. Ser. 192, 14 (2011).

[76] N. Aghanim et al. (Planck Collaboration), arXiv:1807.06209.

[77] M. L. Ahnen et al. (MAGIC, Fermi-LAT Collaborations), J. Cosmol. Astropart. Phys. 02 (2016) 039.

[78] M. Cirelli, G. Corcella, A. Hektor, G. Hutsi, M. Kadastik, P. Panci, M. Raidal, F. Sala, and A. Strumia, J. Cosmol. Astropart. Phys. 03 (2011) 051; 10 (2012) E01.

[79] A. Ibarra, D. Tran, and C. Weniger, Int. J. Mod. Phys. A 28, 1330040 (2013).

[80] J. M. Gaskins, Contemp. Phys. 57, 496 (2016).

[81] T. R. Slatyer, in Proceedings, Theoretical Advanced Study Institute in Elementary Particle Physics: Anticipating the Next Discoveries in Particle Physics (TASI 2016): Boulder, CO, USA, 2016 (2018), pp. 297-353.

[82] D. Hooper, Proc. Sci. TASI2018 (2019) 010. 
[83] J. F. Navarro, A. Ludlow, V. Springel, J. Wang, M. Vogelsberger, S. D. M. White, A. Jenkins, C. S. Frenk, and A. Helmi, Mon. Not. R. Astron. Soc. 402, 21 (2010).

[84] A. Airapetian et al. (HERMES Collaboration), Phys. Rev. D 75, 012007 (2007).

[85] E. S. Ageev et al. (Compass Collaboration), Phys. Lett. B 647, 330 (2007).

[86] D. S. Akerib et al. (LUX Collaboration), Phys. Rev. Lett. 116, 161302 (2016).

[87] D. S. Akerib et al. (LUX-ZEPLIN Collaboration), Phys. Rev. D 101, 052002 (2020).

[88] R. W. Schnee, in Physics of the large and the small, TASI 09, Proceedings of the Theoretical Advanced Study Institute in Elementary Particle Physics, Boulder, Colorado, USA, 2009 (2011), pp. 775-829.

[89] J. Alwall, M. Herquet, F. Maltoni, O. Mattelaer, and T. Stelzer, J. High Energy Phys. 06 (2011) 128.

[90] J. Alwall, R. Frederix, S. Frixione, V. Hirschi, F. Maltoni, O. Mattelaer, H. S. Shao, T. Stelzer, P. Torrielli, and M. Zaro, J. High Energy Phys. 07 (2014) 079.

[91] R. Placakyte (H1and for the ZEUS Collaboration), in Proceedings, 31st International Conference on Physics in collisions (PIC 2011): Vancouver, Canada, 2011 (2011).

[92] R. D. Ball et al., Nucl. Phys. B867, 244 (2013).

[93] R. D. Ball et al. (NNPDF Collaboration), J. High Energy Phys. 04 (2015) 040. 